Pacific Journal of Mathematics

INCIDENCE MATRICES AND INTERVAL GRAPHS 


\title{
INCIDENCE MATRICES AND INTERVAL GRAPHS
}

\author{
D. R. Fulkerson and O. A. Gross
}

According to present genetic theory, the fine structure of genes consists of linearly ordered elements. A mutant gene is obtained by alteration of some connected portion of this structure. By examining data obtained from suitable experiments, it can be determined whether or not the blemished portions of two mutant genes intersect or not, and thus intersection data for a large number of mutants can be represented as an undirected graph. If this graph is an "interval graph," then the observed data is consistent with a linear model of the gene.

The problem of determining when a graph is an interval graph is a special case of the following problem concerning $(0,1)$-matrices: When can the rows of such a matrix be permuted so as to make the 1's in each column appear consecutively? A complete theory is obtained for this latter problem, culminating in a decomposition theorem which leads to a rapid algorithm for deciding the question, and for constructing the desired permutation when one exists.

Let $A=\left(a_{i j}\right)$ be an $m$ by $n$ matrix whose entries $\alpha_{i j}$ are all either 0 or 1 . The matrix $A$ may be regarded as the incidence matrix of elements $e_{1}, e_{2}, \cdots, e_{m}$ vs. sets $S_{1}, S_{2}, \cdots, S_{n}$; that is, $a_{i j}=0$ or 1 according as $e_{i}$ is not or is a member of $S_{j}$. For certain applications, one of which will be discussed below, it is of interest to know whether or not one can order the elements in such a way that each set $S_{j}$ consists of elements that appear consecutively in the ordering. In terms of the incidence matrix $A$, the question is whether there is an $m$ by $m$ permutation matrix $P$ such that the 1's in each column of PA occur in consecutive positions. We shall describe a computationally efficient method of answering this question, and of determining such a $P$ when one exists.

Given a family of sets $S_{1}, S_{2}, \cdots, S_{n}$, one can form the intersection graph of the family by associating a vertex of the graph with each set and joining two distinct vertices with an edge if their corresponding sets have a nonempty intersection. Conversely, any finite graph can of course be viewed as the intersection graph of a family of sets (in many ways). If each set can be taken as an interval on the real line, the graph is called an interval graph. Interval graphs have been investigated in $[7,5,3]$. The problem posed above is closely related to that of determining whether a given graph is an interval

Received April 21, 1964. 
graph. Necessary and sufficient conditions for this are known $[7,3]$. But our problem appears to be more general, and our approach is quite different.

The study of interval graphs was stimulated in part by a biological application concerning the fine structure of genes [1, 7]. A basic problem, posed in [1], is to decide whether or not the subelements of genes are linked together in a linear order. A way of approaching this problem is also described in [1]. Briefly, it is as follows. For certain microorganisms, there are a standard form and mutants, the latter arising from the standard form by alteration of some connected part of the genetic structure. Experiments can be devised for determining whether the blemished parts of two mutant genes intersect or not. Thus the mathematical problem becomes: Given a large number of mutants together with information as to when the blemished portions of pairs of mutants intersect, to decide whether this information is compatible with a linear model of the gene or not. One example, analyzed in [1], shows intersection data for 145 mutants of a certain virus, for which it was found that a linear model was adequate.

In attacking the combinatorial problem posed at the beginning of this section, it does not suffice to consider just the intersection graph of the sets $S_{1}, S_{2}, \cdots, S_{n}$. Instead, we focus attention primarily on a more restricted graph, the overlap graph (§3), two sets being said here to overlap if they have a nonempty intersection which is properly included in each. The connected components of the overlap graph partition the $m$ by $n$ incidence matrix $A$ into $m$-rowed submatrices $A_{1}, A_{2}, \cdots, A_{p}$. Thus we can take $A=\left(A_{1}, A_{2}, \cdots, A_{p}\right)$, where each $A_{k}$ corresponds to a component of the overlap graph. Then our main theorem (Theorem 4.1) asserts that if there are permutations $P_{k}, k=$ $1,2, \cdots, p$, such that $P_{k} A_{k}$ has consecutive 1 's in each column, there is a permutation $P$ such that $P A$ has this property also. This somewhat surprising result, coupled with the fact that one can describe a simple and direct construction $(\$ 5)$ for testing whether such $P_{k}$ exist, provides an answer to the existence question for a general incidence matrix $A$.

2. A basic theorem. Let $A$ be a $(0,1)$-matrix. We say that $A$ has the consecutive 1's property (for columns) provided there is a permutation matrix $P$ such that the 1's in each column of $P A$ occur consecutively. We note to begin with that the intersection graph of $A$ does not contain enough information to decide whether $A$ has the property or not. For example, the matrices

$$
A_{1}=\left[\begin{array}{lll}
1 & 1 & 1 \\
0 & 0 & 0 \\
0 & 0 & 0
\end{array}\right], \quad A_{2}=\left[\begin{array}{lll}
0 & 1 & 1 \\
1 & 0 & 1 \\
1 & 1 & 0
\end{array}\right]
$$


have the same intersection graph, a triangle, but $A_{2}$ does not have the consecutive 1's property. The first question that naturally comes up, then, is how much information about $A$ is needed to decide whether it has the property or not. Do we need to know $A$ itself, or will something less suffice? Theorem 2.1 below provides a partial answer to this question; it shows that a knowledge of the matrix $A^{T} A$ is enough. Here $A^{T}$ denotes the transpose of $A$.

\section{THEOREM 2.1. Let $A$ and $B$ be $(0,1)$-matrices satisfying}

$$
A^{T} A=B^{T} B \text {. }
$$

Then either both $A$ and $B$ have the consecutive 1's property or neither does. Moreover, if $A$ and $B$ have the same number of rows and $A$ has the consecutive 1's property, then there is a permutation $P$ such that $B=P A$.

Proof. The first part of the theorem follows trivially from the second. For assume (2.1) and let $A$ be $m$ by $n, B$ be $m^{\prime}$ by $n$, with $m>m^{\prime}$. Then we may adjoin $m-m^{\prime}$ rows of 0 's to $B$, thereby obtaining an $m$ by $n$ matrix $C$ satisfying $A^{T} A=C^{T} C$. The second assertion of the theorem then implies $C=P A$, for some permutation $P$. Consequently both $A$ and $C$ (hence both $A$ and $B$ ) have the property or neither does.

Let $A$ and $B$ be $m$ by $n$. The second part of the theorem is obviously valid for $n=1$, and we proceed by induction on $n$. Suppress the first column vector $a$ of $A$ and the first column vector $b$ of $B$, and call the resulting matrices $A_{1}$ and $B_{1}$. Thus

$$
\begin{aligned}
& A=\left(a, A_{1}\right), \\
& B=\left(b, B_{1}\right) .
\end{aligned}
$$

Clearly $A_{1}$ has the consecutive 1's property and $A_{1}^{T} A_{1}=B_{1}^{T} B_{1}$. Hence, by the induction hypothesis, we can permute the rows of $B$ to obtain a matrix $\widetilde{B}$ satisfying

$$
\widetilde{B}=\left(\widetilde{b}, A_{1}\right),
$$

the column vector $\widetilde{b}$ being a permutation of $b$.

We shall finish the proof by showing that, corresponding to each row vector of $\widetilde{B}$, there is an equal row vector of $A$. We begin by noting that if the column vectors $a$ of (2.2) and $\tilde{b}$ of (2.4) have common 1's (common 0's), the corresponding rows of $A$ and $\widetilde{B}$ are equal and can be paired off against each other. Having done this, consider the submatrices of $A$ and $\widetilde{B}$ consisting of the remaining rows. Call these matrices $A^{*}$ and $B^{*}$. Thus we may write 


$$
\begin{aligned}
& A^{*}=\left(a^{*}, A_{1}^{*}\right), \\
& B^{*}=\left(b^{*}, A_{1}^{*}\right) .
\end{aligned}
$$

Note that $A^{*}$ inherits the consecutive 1's property from $A$, since suppression of rows does not destroy the property, and hence we can permute the rows of $A^{*}$ to obtain a matrix

$$
\bar{A}=\left(\bar{a}, \bar{A}_{1}\right)
$$

in which the 1's in each column occur consecutively. Applying the same permutation to $B^{*}$ yields

$$
\bar{B}=\left(\bar{b}, \bar{A}_{1}\right) .
$$

Now $\bar{a}$ and $\bar{b}$ are complementary $(0,1)$-vectors, that is, $\bar{b}$ is obtained from $\bar{a}$ by interchanging 0 's and 1's. We also have

$$
\bar{A}^{T} \bar{A}=\bar{B}^{T} \bar{B},
$$

since $\bar{A}$ and $\bar{B}$ are obtained from $A$ and $B$, respectively, by suppressing certain equal rows and permuting rows. In particular, calculating the inner product of $\bar{a}$ with itself and $\bar{b}$ with itself, we have

$$
\bar{a} \cdot \bar{a}=\bar{b} \cdot \bar{b},
$$

and hence $\bar{a}$ and $\bar{b}$ have the same number of 1's (hence also of 0 's), so that the number of 1 's in $\bar{a}$ is equal to the number of 0 's in $\bar{a}$. Thus $\bar{A}$ and $\bar{B}$ have the following appearance:

$$
\bar{A}=\left[\begin{array}{c|c}
0 & \\
\vdots & E \\
0 & \\
\hline 1 & \\
\vdots & F \\
1 & \\
\hline 0 & \\
\vdots & G \\
0 &
\end{array}\right],
$$$$
\bar{B}=\left[\begin{array}{c|c}
1 & \\
\vdots & E \\
1 & \\
\hline 0 & \\
\vdots & F \\
0 & \\
\hline 1 & \\
\vdots & G \\
1 &
\end{array}\right] .
$$

The matrices $E$ and $G$ in (2.11) have together the same number of rows that $F$ does, and we shall show that the rows of $\bar{A}$ corresponding to $F$ (corresponding to $E$ and $G$ ) can be paired with equal rows of $\bar{B}$ corresponding to $E$ and $G$ (corresponding to $F$ ).

To this end, let $E$ and $G$ have $k$ and $l$ rows, respectively, and let $R_{p}$ denote the $p$ by $p$ permutation matrix which reverses order, 


$$
R_{p}=\left[\begin{array}{ccc}
0 & \therefore & 1 \\
1 & & 0
\end{array}\right]
$$

We assert that

$$
F=\left[\begin{array}{l}
R_{k} E \\
R_{l} G
\end{array}\right]
$$

For consider an arbitrary column vector $c$ of $\bar{A}_{1}$ and write

$$
c=\left[\begin{array}{l}
e \\
f \\
g
\end{array}\right],
$$

corresponding to the partition (2.11) of $\bar{A}_{1}$. From (2.9) we have

$$
\bar{a} \cdot c=\bar{b} \cdot c,
$$

and thus the number of 1's in $f$ is equal to the number of 1 's in $e$ and $g$. It follows from this and the fact that the 1's in $c$ occur consecutively that

$$
f=\left[\begin{array}{l}
R_{k} e \\
R_{l} g
\end{array}\right] .
$$

This establishes (2.13) and finishes the proof of Theorem 2.1.

We note the following corollary of Theorem 2.1.

Corollary 2.2. Let $A$ and $B$ be $(0,1)$-matrices satisfying (2.1). If $A$ has the consecutive 1's property and has no rows of 0 's, then there is a permutation $P$ such that

$$
P B=\left[\begin{array}{c}
A \\
0
\end{array}\right] \text {. }
$$

Proof. Let $A$ be $m$ by $n$ and let $B$ be $m^{\prime}$ by $n$. Suppose $m^{\prime}<m$. Then we may adjoin $m-m^{\prime}$ rows of 0 's to $B$, obtaining a matrix $C$ satisfying $A^{T} A=C^{T} C$. By Theorem 2.1, $C$ is a row permutation of $A$, violating the assumption that $A$ has no zero rows. Consequently $m^{\prime} \geqq m$. The conclusion now follows by adjoining $m^{\prime}-m$ rows of 0 's to $A$.

It is of course not true in general that equation (2.1) implies $B=P A$ for $m$ by $n(0,1)$-matrices $A$ and $B$. A simple example is

$$
A=\left[\begin{array}{lll}
1 & 1 & 1 \\
1 & 0 & 0 \\
0 & 1 & 0 \\
0 & 0 & 1
\end{array}\right], \quad B=\left[\begin{array}{lll}
0 & 1 & 1 \\
1 & 0 & 1 \\
1 & 1 & 0 \\
0 & 0 & 0
\end{array}\right] \text {. }
$$


It would be interesting to know conditions on $A^{T} A$ in order that $A$ have the consecutive 1's property. Although we have not been able to give a complete answer to this question in the sense of finding some nice set of necessary and sufficient conditions, we shall establish a theorem in $\S 4$ which reduces the question to the consideration of $(0,1)$-matrices having connected overlap graphs. Section 5 then provides a simple construction for testing whether or not such a matrix has the property.

3. The overlap graph and component graph. We rephrase the definition of "overlap" in terms of inner products of $(0,1)$-vectors. Let $a$ and $b$ be $(0,1)$-vectors having $m$ components. Their inner product $a \cdot b$ satisfies

$$
0 \leqq a \cdot b \leqq \min (a \cdot a, b \cdot b) .
$$

If strict inequality holds throughout (3.1), that is, if $a \cdot b$ satisfies

$$
0<a \cdot b<\min (a \cdot a, b \cdot b) \text {, }
$$

we say that $a$ and $b$ overlap. We also say that $a$ and $b$ are disjoint if

$$
a \cdot b=0 \text {, }
$$

and that $a$ contains $b$ if

$$
a \cdot b=b \cdot b \text {. }
$$

Now let $A$ be an $m$ by $n(0,1)$-matrix having column vectors $a_{1}, a_{2}, \cdots, a_{n}$. It is convenient, and imposes no loss of generality in studying the consecutive 1's property, to assume that $a_{j} \neq 0, j=$ $1,2, \cdots, n$, and that $a_{i} \neq a_{j}$ for $i \neq j$. Henceforth we frequently make these assumptions and refer to such an $A$ as proper.

There are various graphs one can associate with a $(0,1)$-matrix $A$ that are meaningful insofar as the consecutive 1's property is concerned. For instance, we can take vertices $x_{1}, x_{2}, \cdots, x_{n}$ corresponding to the columns $a_{1}, a_{2}, \cdots, a_{n}$ of $A$, and put in the following edges, some being directed, others undirected: an edge $\left[x_{i}, x_{j}\right]$ directed from $x_{i}$ to $x_{j}$ if $a_{i}$ contains $a_{j}$; an undirected edge $\left(x_{i}, x_{j}\right)$ joining $x_{i}$ and $x_{j}$ if $a_{i}$ and $a_{j}$ overlap. (If we go on to make this a "weighted" graph by associating with each edge (vertex) the appropriate inner product, then Theorem 2.1 shows that we have included sufficient information to decide whether $A$ has the consecutive 1's property). We shall not, however, deal primarily with this full graph $\mathscr{F}=\mathscr{F}(A)$, but shall instead work mostly with two graphs derived from it, one of these being an undirected graph, the other a directed graph.

The first of these is the graph obtained from $\mathscr{F}$ by including only 
the undirected edges $\left(x_{i}, x_{j}\right)$ corresponding to overlapping column vectors $a_{i}$ and $a_{j}$. We call this the overlap graph of $A$ and denote it by $\mathscr{G}=\mathscr{G}(A)$.

The second of these graphs is obtained from $\mathscr{F}$ as follows: Let $X_{1}, X_{2}, \cdots, X_{p}$ be the connected components of $\mathscr{C}(A)$, considered as vertices of a graph $\mathscr{D}=\mathscr{D}(A)$. Direct an edge $\left[X_{i}, X_{j}\right]$ from vertex $X_{i}$ to vertex $X_{j}$ if there is an $x$ in $X_{i}$ and a $y$ in $X_{j}$ such that $[x, y]$ is an edge from $x$ to $y$ in $\mathscr{F}$. We call $\mathscr{D}$ the component graph of $A$. The component graph $\mathscr{D}$ is obtained by condensing in $\mathscr{F}$ the vertices of a connected component of $\mathscr{G}$ to a single vertex.

We give an example illustrating these concepts. Let

Thus

$$
A=\left[\begin{array}{llllllll}
1 & 0 & 0 & 0 & 0 & 0 & 0 & 0 \\
1 & 1 & 1 & 0 & 0 & 0 & 1 & 0 \\
0 & 1 & 0 & 1 & 1 & 0 & 0 & 0 \\
1 & 1 & 1 & 0 & 0 & 1 & 0 & 1 \\
1 & 1 & 1 & 0 & 0 & 0 & 0 & 1 \\
0 & 1 & 0 & 0 & 1 & 0 & 0 & 0 \\
1 & 1 & 1 & 0 & 0 & 1 & 1 & 0 \\
0 & 1 & 0 & 1 & 0 & 0 & 0 & 0 \\
1 & 1 & 1 & 0 & 0 & 0 & 0 & 1
\end{array}\right] .
$$

$$
A^{T} A=\left[\begin{array}{llllllll}
6 & 5 & 5 & 0 & 0 & 2 & 2 & 3 \\
5 & 8 & 5 & 2 & 2 & 2 & 2 & 3 \\
5 & 5 & 5 & 0 & 0 & 2 & 2 & 3 \\
0 & 2 & 0 & 2 & 1 & 0 & 0 & 0 \\
0 & 2 & 0 & 1 & 2 & 0 & 0 & 0 \\
2 & 2 & 2 & 0 & 0 & 2 & 1 & 1 \\
2 & 2 & 2 & 0 & 0 & 1 & 2 & 0 \\
3 & 3 & 3 & 0 & 0 & 1 & 0 & 3
\end{array}\right] .
$$

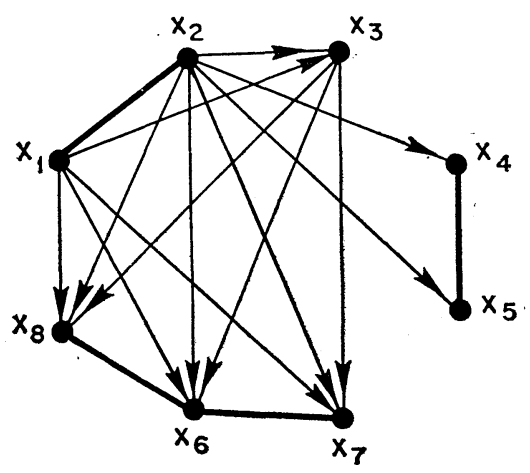

Fig. 3.1. The full graph $\mathscr{F}(A)$

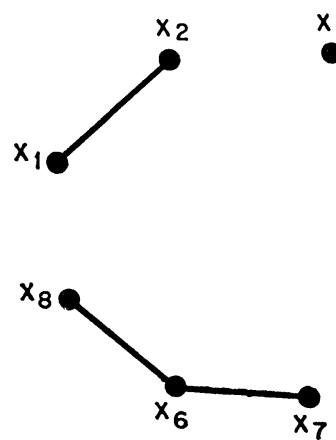

Fig. 3.2. The overlap graph $\mathscr{G}(A)$ 


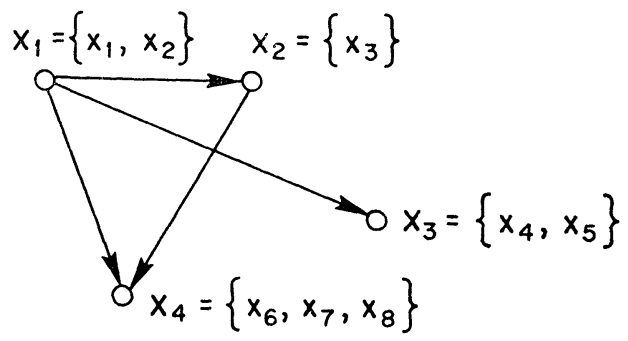

Fig. 3.3. The component graph $\mathscr{D}(A)$

The graphs $\mathscr{F}, \mathscr{G}$, and $\mathscr{D}$ for $A$ are shown in Figs. 3.1, 3.2, and 3.3 , respectively.

Notice in the example that the component graph $\mathscr{D}$ is acyclic (has no directed cycles) and transitive (if $[X, Y]$ and $[Y, Z]$ are edges, then $[X, Z]$ is an edge). We now prove that this is generally so.

Theorem 3.1. The component graph $\mathscr{D}(A)$ of a proper $(0,1)$ matrix $A$ is acyclic and transitive.

Proof. We first show that $\mathscr{D}$ is transitive. Let $X, Y, Z$ be components of $\mathscr{G}$ (vertices of $\mathscr{D}$ ) such that $[X, Y]$ and $[Y, Z]$ are edges in $\mathscr{D}$. Hence there are vertices $x, y, y^{\prime}, z$ of $\mathscr{F}$ with $x$ in $X, y$ and $y^{\prime}$ in $Y$, and $z$ in $Z$, such that $[x, y]$ and $\left[y^{\prime}, z\right]$ are directed edges of $\mathscr{F}$. Moreover, since $y$ and $y^{\prime}$ are in the same component of $\mathscr{G}$, there is a chain $\left(y, y_{1}\right),\left(y_{1}, y_{2}\right), \cdots,\left(y_{k}, y^{\prime}\right)$ of undirected edges joining $y$ to $y^{\prime}$ in $\mathscr{G}$ (hence in $\mathscr{F}$ ). Thus the matrix $A$ has corresponding columns $a, b, b^{\prime}, c$ and $b_{1}, b_{2}, \cdots, b_{k}$ such that $a$ contains $b, b^{\prime}$ contains $c, b$ and $b_{1}$ overlap, $b_{1}$ and $b_{2}$ overlap, $\cdots, b_{k}$ and $b^{\prime}$ overlap. But if $a$ contains $b$ and $b$ overlaps $b_{1}$, then either $a$ contains $b_{1}$ or $a$ overlaps $b_{1}$. The latter of these alternatives is impossible, for otherwise $x$ and $y_{1}$ would be in the same component of the overlap graph, contrary to choice. Thus $a$ contains $b_{1}$. We may now repeat the argument with $a, b_{1}, b_{2}$ in place of $a, b, b_{1}$ to show that $a$ contains $b_{2}$, and so on, finally deducing that $a$ contains $b^{\prime}$. Since $b^{\prime}$ contains $c$, we see that $a$ contains $c$, and hence $[x, z]$ is a directed edge in $\mathscr{F}$. Consequently $[X, Z]$ is a directed edge in $\mathscr{D}$, and $\mathscr{D}$ is transitive.

To show that $\mathscr{D}$ is acyclic, it suffices, since $\mathscr{D}$ is transitive, to show that both $[X, Y]$ and $[Y, X]$ cannot be edges of $\mathscr{D}$. Suppose they were edges of $\mathscr{D}$. Then, as above, the matrix $A$ would have columns $a_{1}, a_{2}, \cdots, a_{k}$ and $b_{1}, b_{2}, \cdots, b_{l}$ with the following relations holding: $a_{1}$ contains $b_{1} ; b_{l}$ contains $a_{k} ; a_{1}$ overlaps $a_{2}, \cdots, a_{k-1}$ overlaps $a_{k}$; $b_{1}$ overlaps $b_{2}, \cdots, b_{l-1}$ overlaps $b_{l}$; and no $a_{i}$ overlaps any $b_{j}$. It then follows as above that $a_{1}$ contains $b_{l}$. Similarly we deduce that $b_{l}$ contains $a_{1}$. Thus $a_{1}=b_{l}$. Since $a_{1}$ and $b_{l}$ are necessarily distinct 
columns of $A$, this violates our assumption that $A$ has no pair of equal columns. Consequently $\mathscr{D}$ is acyclic. This completes the proof of Theorem 3.1.

Theorem 3.1 shows that $\mathscr{D}$ is the graph of a partial ordering. This partial ordering is special in the sense that an element can have at most one immediate predecessor, as Theorem 3.2 below shows. Consequently, if we omit from $\mathscr{D}$ every edge whose existence is implied by transitivity, the resulting graph is simply a collection of rooted trees. (For example, in Fig. 3.3, omitting the edge $\left[X_{1}, X_{4}\right]$ produces a single tree with root $X_{1}$ ).

THEOREM 3.2. Each vertex of the component graph $\mathscr{D}(A)$ of a proper $(0,1)$-matrix $A$ has at most one immediate predecessor.

Proof. It suffices to show that if $[X, Z]$ and $[Y, Z]$ are edges of $\mathscr{D}$, then either $[X, Y]$ or $[Y, X]$ is an edge. Thus, let $[X, Z]$ and $[Y, Z]$ be edges of $\mathscr{D}$. It follows that $A$ has columns $a, b, c_{1}, c_{2}, \cdots$, $c_{k}$ with the following relations holding: $a$ contains $c_{1}, b$ contains $c_{k}$, and successive pairs of $c$ 's overlap. Thus $a$ contains $c_{k}$. Since $b$ also contains $c_{k}$ and $a$ and $b$ do not overlap, then either $a$ contains $b$ or $b$ contains $a$. Consequently, either $[X, Y]$ or $[Y, X]$ is an edge of $\mathscr{D}$.

In $\S 4$ we shall use the structure of the component graph to prove the decomposition theorem mentioned in $\S 1$. This structure will also be exploited later in developing a complete algorithm for arranging the rows of a matrix $A$ to make its 1's appear consecutively in each column, when this is possible. For these purposes, we note here another fact about the full graph $\mathscr{F}(A)$ that has not been stated explicitly, although its proof is contained in that of Theorem 3.1.

THEOREM 3.3. Let $X$ and $Y$ be components of the overlap graph $\mathscr{G}(A)$ of a proper $(0,1)$-matrix $A$, such that there is an $x$ in $X$ and $y$ in $Y$ with $[x, y]$ an edge of $\mathscr{F}(A)$. Then $\left[x, y^{\prime}\right]$ is an edge of $\mathscr{F}(A)$ for arbitrary $y^{\prime}$ in $Y$.

Theorem 3.3 shows that each column of $A$ corresponding to $Y$ plays exactly the same role with respect to columns of $A$ corresponding to $X$. That is, each " $Y$-column" will be contained in certain of the " $X$-columns" and disjoint from the others. This pattern is the same for every $Y$-column.

4. The decomposition theorem. For an arbitrary $(0,1)$-matrix $A$, we can rearrange columns if necessary and write

$$
A=\left(A_{1}, A_{2}, \cdots, A_{p}\right),
$$


where each submatrix $A_{k}, k=1,2, \cdots, p$, corresponds to a component $X_{k}$ of the overlap graph $\mathscr{G}(A)$. We term (4.1) an overlap decomposition of $A$. It is of course unique, apart from the ordering of the submatrices. We refer to these submatrices as overlap components of $A$, or briefly, components of $A$. If $A$ has just one component, we say that $A$ is connected.

THEOREM 4.1. A (0,1)-matrix $A$ has the consecutive 1's property if and only if each of its components has the property.

Proof. The necessity is obvious. To prove sufficiency, we may assume that $A$ is proper and proceed by induction on the number $p$ of components of $A$, the case $p=1$ being trivial.

Before going to the induction step, we first remark that if a matrix has the consecutive 1's property and has equal rows, such rows can be brought together in a permuted form which makes the 1's in each column consecutive. To see this, it suffices to observe two things. First, if a row is deleted from a matrix having the consecutive 1's property, the resulting matrix has the property. On the other hand, if a deleted row is one of a pair of equal rows, then it can be reinserted adjacent to its counterpart in the permuted matrix, and the 1's in each column are still consecutive.

To establish the induction step, let $A$ be a proper matrix having $p$ components, each of which has the consecutive 1's property, and write

$$
A=\left(A_{1}, A_{2}, \cdots, A_{p}\right),
$$

where $A_{p}$ corresponds to a minimal element in the partial ordering given by $\mathscr{D}(A)$. Now delete $A_{p}$ from $A$. By the induction assumption, the matrix

$$
A^{\prime}=\left(A_{1}, A_{2}, \cdots, A_{p-1}\right)
$$

has the consecutive 1's property, and hence, by the above remark, there is a permutation $P$ such that $P A^{\prime}$ has consecutive 1 's in each column and like rows of $P A^{\prime}$ occur together. Consider

$$
P A=\left(B_{1}, B_{2}, \cdots, B_{p}\right)=B .
$$

We may select a topmost and bottommost 1 in $B_{p}$, and write

$$
B=\left[\begin{array}{ccccc}
* & * & \cdots & * & 0 \\
\bar{B}_{1} & \bar{B}_{2} & \cdots & \bar{B}_{p-1} & \bar{B}_{p} \\
* & * & \cdots & * & 0
\end{array}\right],
$$


where the first and last rows of $\bar{B}_{p}$ each contain at least one 1 . Now consider the matrix

$$
\left(\bar{B}_{1}, \bar{B}_{2}, \cdots, \bar{B}_{p-1}\right) \text {. }
$$

We assert that all rows of (4.6) are identical. To see this, observe first that since $X_{p}$ is a minimal element in the partial ordering, each $X_{k}, k=1,2, \cdots, p-1$, either dominates $X_{p}$ (there is an edge from $X_{k}$ to $X_{p}$ in $\mathscr{D}(A)$ ) or is unrelated to $X_{p}$ (there is no edge from $X_{k}$ to $X_{p}$ or from $X_{p}$ to $X_{k}$ ). Suppose $X_{k}$ is unrelated to $X_{p}$. Then, since the first and last rows of $\bar{B}_{p}$ contain a 1 , it follows that the first and last rows of $\bar{B}_{k}$ are all 0 's, for otherwise there would be a column of $A_{p}$ and a column of $A_{k}$ which are not disjoint. On the other hand, suppose $X_{k}$ dominates $X_{p}$. It then follows from Theorem 3.3 that the first and last rows of $\bar{B}_{k}$ are equal. Hence the first and last rows of (4.6) are equal, and consequently, by the selection of $P$, all rows of (4.6) are equal.

We may now permute the rows of $\bar{B}_{p}$ to make its 1 's consecutive in each column. Such a permutation merely shuffles like rows in (4.5), and thus $A$ has the consecutive 1's property.

Any component of $A$ which has no more than two columns obviously has the consecutive 1's property. We may thus state the following corollary of Theorem 4.1.

Corollary 4.2. If each component of a $(0,1)$-matrix $A$ has at most two columns, then $A$ has the consecutive 1's property.

5. Testing a connected matrix for the consecutive 1's property. In this section we shall describe a construction for deciding whether a connected matrix $A$ has the consecutive 1's property. The essential idea is not to search explicitly for a permutation $P$ which rearranges $A$, but rather to attempt to build up a permuted form of $A$, column by column, imposing necessary inner-product requirements on the columns of the configuration.

Let $a, b, c$ be distinct column vectors of $A$ such that $a$ overlaps $b$ and $b$ overlaps $c$. If $A$ has the consecutive 1's property, then there is a row permutation of $A$ which contains the configuration $C_{1}$ or $C_{2}$ described below (and illustrated in Figs. 5.1 and 5.2) according as

$$
a \cdot c<\min (a \cdot b, b \cdot c)
$$

or

$$
a \cdot c \geqq \min (a \cdot b, b \cdot c)
$$

holds. The configuration $C_{1}$ is obtained by writing down $a \cdot a 1$ 's con- 
secutively in a column, then $b \cdot b$ consecutive 1 's in a second column so that these two strings of 1's overlap (at the bottom of the first, say) on $a \cdot b 1$ 's, then a third string of $c \cdot c 1$ 's, overlapping the second string in the same way that the second overlapped the first (at its bottom) on $b \cdot c$ 1's. The configuration $C_{2}$ differs from $C_{1}$ only by making the third string of 1's overlap the second opposite to the manner in which the second overlapped the first.

$\begin{array}{llllll}a & b & c & a & b & c \\ 1 & & & 1 & & 1 \\ 1 & 1 & & 1 & 1 & 1 \\ 1 & 1 & & 1 & 1 & 1 \\ 1 & 1 & & 1 & 1 & 1 \\ 1 & 1 & 1 & 1 & 1 & \\ & 1 & 1 & & 1 & \\ & 1 & 1 & & 1 & \end{array}$

Fig. 5.1. The Configuration $C_{1}$

Fig. 5.2. The Configuration $C_{2}$

Note that while we have two choices in placing the second string of 1's relative to the first (top or bottom of the first), it is immaterial which of these we select. Moreover, having selected one, there is no further latitude in positioning the third string of 1 's, since its position relative to the second is determined by (5.1) or (5.2) and the position of the second relative to the first. After constructing the configuration $C_{1}$ or $C_{2}$, we can then check the inner product of its first and third columns. If this is not equal to $a \cdot c$, we know immediately that $A$ does not have the consecutive 1's property.

Three column vectors of $A$ which can be ordered so that the first and second overlap and the second and third overlap will be termed a rigid triple of $A$. Our construction for a connected $A$ will be based on singling out a spanning subtree of $\mathscr{G}(A)$ and then positioning strings of 1's, using rigid triples obtained from this tree.

Instead of describing the computation formally, we shall consider an example which will make the process clear. Let

$$
A=\left[\begin{array}{llllll}
1 & 0 & 0 & 1 & 1 & 0 \\
0 & 0 & 1 & 0 & 0 & 1 \\
1 & 1 & 1 & 1 & 0 & 1 \\
0 & 0 & 0 & 0 & 1 & 0 \\
1 & 1 & 1 & 1 & 1 & 0 \\
1 & 0 & 0 & 0 & 1 & 0
\end{array}\right] .
$$


A spanning subtree of the overlap graph $\mathscr{G}(A)$ is shown in Fig. 5.3 below.

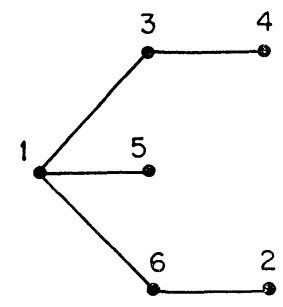

Fig. 5.3. A Spanning Subtree of $\mathscr{G}(A)$

On the first step, we may position the rigid triple $1,3,5$ to obtain the configuration of Fig. 5.4 below.

$\begin{array}{lll}\text { (1) } & \text { (3) } & \text { (5) } \\ & & 1 \\ 1 & & 1 \\ 1 & & 1 \\ 1 & 1 & 1 \\ 1 & 1 & \\ & 1 & \end{array}$

Fig. 5.4

The inner products of this configuration agree with the corresponding ones of $A$, and we proceed to add column 6 , positioning it relative to 1 and 5 (5, 1, 6 form a rigid triple), to obtain the configuration shown in Fig. 5.5.

$\begin{array}{llll}\text { (1) } & \text { (3) } & \text { (5) } & \text { (6) } \\ & & 1 & \\ 1 & & 1 & \\ 1 & & 1 & \\ 1 & 1 & 1 & \\ 1 & 1 & & 1 \\ & 1 & & 1\end{array}$

Fig. 5.5

Checking the inner product of column 6 with all others in the configuration shows that we have no contradiction yet, and so we add column 4 , positioning it relative to 1 and 3 , to obtain the configuration of Fig. 5.6. 


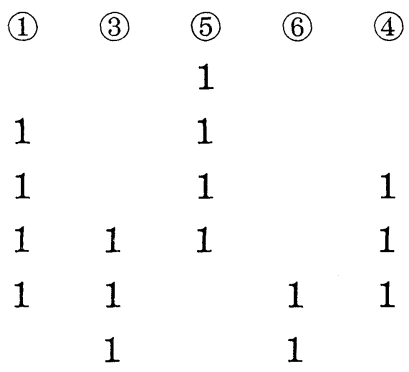

Fig. 5.6

Again all inner products of column 4 with preceding columns of the configuration check out properly. We then add column 2, positioning it relative to 1 and 6 , obtaining the configuration of Fig. 5.7.

$\begin{array}{llllll}\text { (1) } & \text { (3) } & \text { (5) } & \text { (6) } & \text { (4) } & \text { (2) } \\ & & 1 & & & \\ 1 & & 1 & & & \\ 1 & & 1 & & 1 & \\ 1 & 1 & 1 & & 1 & 1 \\ 1 & 1 & & 1 & 1 & 1 \\ & 1 & & 1 & & \end{array}$

Fig. 5.7

Since the inner products of column 2 with all preceding columns in the configuration of Fig. 5.7 agree with the corresponding inner products of columns of $A$, Corollary 2.2 shown that the matrix

$$
B=\left[\begin{array}{llllll}
1 & 2 & 3 & 4 & 5 & 6 \\
0 & 0 & 0 & 0 & 1 & 0 \\
1 & 0 & 0 & 0 & 1 & 0 \\
1 & 0 & 0 & 1 & 1 & 0 \\
1 & 1 & 1 & 1 & 1 & 0 \\
1 & 1 & 1 & 1 & 0 & 1 \\
0 & 0 & 1 & 0 & 0 & 1
\end{array}\right],
$$

obtained from Fig. 5.7 by rearranging columns and filling in 0 's so that $B$ has no zero rows, is a row permutation of $A$.

A complete test for a general matrix $A$ is now clear. First determine the connected components of $A$. This can be easily done in such a way that corresponding spanning subtrees are automatically obtained. Simply select an arbitrary column and find all columns it overlaps; then select one of these and determine any new columns it overlaps, and so on. When no new columns can be determined, a spanning subtree for 
one component has been found. The configuration building process described above can then be applied to each component. (In actual computation, one would build the configuration simultaneously with the determination of a connected component.)

If one measures the efficiency of this method by calculating an upper bound on the number of times that it is necessary to compute (or look up) the inner product of two $(0,1)$-vectors, it is not difficult to see that if $A$ has $n$ columns, such a bound is of the order $O\left(n^{2}\right)$.

6. Fitting the components together. If no contradiction in inner products is encountered in the construction outlined in the preceding section, one can go on to find the desired row permutation of $A$ by fitting the various connected submatrices together in an appropriate way, using the partial ordering of components given by $\mathscr{D}(A)$. (We assume that $A$ is proper.) The proof of Theorem 4.1 indicates how to proceed. Again we shall not describe the process in detail, but shall illustrate it with the example of $\S 3$.

The configuration-building procedure of $\S 5$ produces the matrices

$$
B_{1}=\left[\begin{array}{ll}
1 & 2 \\
1 & 0 \\
1 & 1 \\
1 & 1 \\
1 & 1 \\
1 & 1 \\
1 & 1 \\
0 & 1 \\
0 & 1 \\
0 & 1
\end{array}\right], \quad B_{2}=\left[\begin{array}{l}
3 \\
0 \\
0 \\
0 \\
1 \\
1 \\
1 \\
1 \\
1
\end{array}\right], \quad B_{3}=\left[\begin{array}{ll}
0 \\
0 & 0 \\
0 & 0 \\
0 & 0 \\
0 & 0 \\
0 & 0 \\
0 & 0 \\
1 & 0 \\
1 & 1 \\
0 & 1
\end{array}\right], \quad B_{4}=\left[\begin{array}{lll}
0 \\
0 & 0 & 0 \\
0 & 0 & 0 \\
0 & 0 & 0 \\
0 & 0 & 0 \\
0 & 1 & 0 \\
1 & 1 & 0 \\
1 & 0 & 1 \\
0 & 0 & 1 \\
0 & 0 & 1
\end{array}\right] .
$$
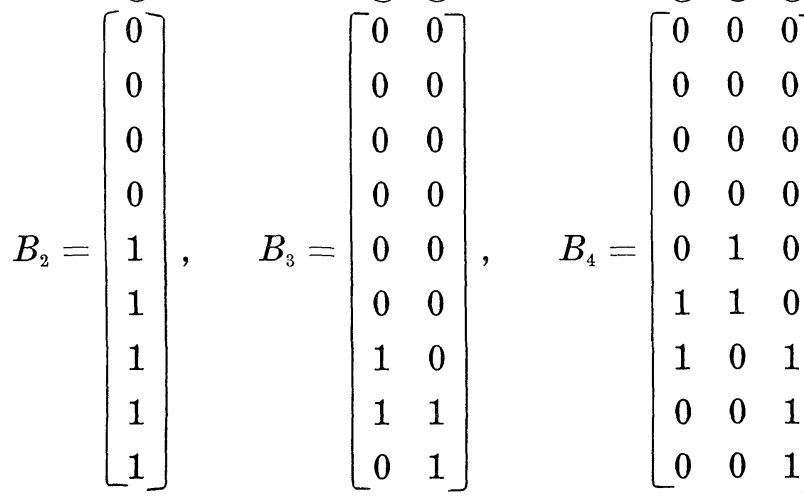

To fit these together, we first determine the maximal elements in $\mathscr{D}(A)$. Here there is just one, corresponding to $B_{1}$, and we have:

(1) (2) (3) (4) (5) (6) (7) (8)

$\left[\begin{array}{ll}1 & 0 \\ 1 & 1 \\ 1 & 1 \\ 1 & 1 \\ 1 & 1 \\ 1 & 1 \\ 0 & 1 \\ 0 & 1 \\ 0 & 1\end{array} \mid\right.$

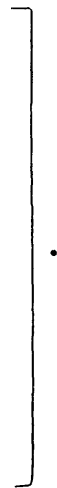


(If there were more than one maximal element, the determined part of the configuration at this point would appear as a direct sum.)

Next suppress the maximal elements in $\mathscr{D}(A)$ and look for the new maximal elements in the reduced graph. Here there are two, corresponding to $B_{2}$ and $B_{3}$. By Theorem 3.3, each column of $B_{2}\left(B_{3}\right)^{\text {s }}$ is contained in certain columns of $B_{1}$ and disjoint from the remaining, and this pattern is the same for all columns of $B_{2}\left(B_{3}\right)$. Hence it suffices to test one column of $B_{2}\left(B_{3}\right)$ to find this pattern. We can do this by referring to $A^{T} A$ or $\mathscr{F}(A)$. The single column of $B_{2}$ is contained in both columns of $B_{1}$, whereas columns of $B_{3}$ are disjoint from the first column of $B_{1}$ and contained in the second. We now fit in $B_{2}$ by sliding its nonzero part (the part between a topmost and bottommost 1 of $B_{2}$ ) to the top of the consecutive group of equal rows of $B_{1}$ consisting of $(1,1)$-pairs. Similarly, we fit in $B_{3}$ by sliding its nonzero part to the top of the consecutive group of $(0,1)$-rows of $B_{1}$. This gives the configuration

\begin{tabular}{|c|c|c|c|c|c|}
\hline 1 & 0 & 0 & 0 & 0 & \\
\hline 1 & 1 & 1 & 0 & 0 & \\
\hline 1 & 1 & 1 & 0 & 0 & \\
\hline 1 & 1 & 1 & 0 & 0 & \\
\hline 1 & 1 & 1 & 0 & 0 & $*$ \\
\hline 1 & 1 & 1 & 0 & 0 & \\
\hline 0 & 1 & 0 & & 0 & \\
\hline 0 & 1 & 0 & 1 & 1 & \\
\hline 0 & 1 & 0 & 0 & 1 & \\
\hline
\end{tabular}

The new reduced component graph consists of the single element corresponding to $B_{4}$, and we see from $\mathscr{F}(A)$ that each column of $B_{4}$ is contained in columns $1,2,3$ and is disjoint from columns 4 and 5 . Consequently we slide the nonzero part of $B_{4}$ to appear at the top of the consecutive group of $(1,1,1,0,0)$-rows in the configuration. This yields a row permutation of $A$ whose 1's appear consecutively in each column:

(1) (2) (3) (4) (5) (6) (7) (8)

$\left[\begin{array}{ll|l|ll|lll}1 & 0 & 0 & 0 & 0 & 0 & 0 & 0 \\ 1 & 1 & 1 & 0 & 0 & 0 & 1 & 0 \\ 1 & 1 & 1 & 0 & 0 & 1 & 1 & 0 \\ 1 & 1 & 1 & 0 & 0 & 1 & 0 & 1 \\ 1 & 1 & 1 & 0 & 0 & 0 & 0 & 1 \\ 1 & 1 & 1 & 0 & 0 & 0 & 0 & 1 \\ 0 & 1 & 0 & 1 & 0 & 0 & 0 & 0 \\ 0 & 1 & 0 & 1 & 1 & 0 & 0 & 0 \\ 0 & 1 & 0 & 0 & 1 & 0 & 0 & 0\end{array}\right]$.


Note that at each stage in solving the example, we automatically had equal rows occurring together (see the proof of Theorem 4.1) and hence did not need to permute rows of the configuration before fitting in another piece. It is not difficult to show inductively that this will always be the case, provided the prescription of sliding the nonzero part of the new piece to the top of the group of like rows which precede it is followed. A relevant fact in making such a proof is that the nonzero part of the new piece contains no zero rows. This follows from connectedness and consecutivity of 1's, and justifies our terminology.

7. Application to interval graphs. The methods developed in preceding sections can be applied to the problem of determining when a graph is an interval graph. As noted in $\S 1$, various sets of necessary and sufficient conditions that a graph be an interval graph are known. Moreover, computational methods based on some of these have been described [7,3]. The procedure we shall outline appears to be more efficient. In addition, a specific representation in terms of intervals can be easily produced if desired.

We begin by reviewing certain concepts and results that will be relevant. The first of these is that of a rigid circuit graph $[2,7]$. An undirected graph ${ }^{1} \mathscr{G}$ is a rigid circuit graph if every circuit of $\mathscr{G}$ with more than three vertices has a chord. Here a chord is an edge not in the circuit which joins two vertices of the circuit. It is easy to see that an interval graph must be a rigid circuit graph [7]. We also recall the following basic result about rigid circuit graphs [2]: Every rigid circuit graph $\mathscr{G}$ has a simplicial vertex. Here a vertex $s$ of $\mathscr{G}$ is simplicial if all the neighbors of $s$ in $\mathscr{G}$ form a simplex or clique in $\mathscr{G}$ (i.e,, each pair of neighbors of $s$ is joined by an edge.) Thus $s$ together with its neighbors is also a clique in $\mathscr{G}$.

Rigid circuit graphs have been characterized in various ways [2, 7]. For our purposes, the following procedure for testing the rigid circuit property will be appropriate: ${ }^{2}$ Search for a simplicial vertex in $\mathscr{G}$; if one is found, supress it and repeat the procedure in the reduced graph. It follows that $\mathscr{G}$ is a rigid circuit graph if and only if this process terminates in the deletion of all vertices of $\mathscr{G}$. For if $\mathscr{G}$ is a rigid circuit graph, then $\mathscr{G}$ contains a simplicial vertex, and deletion of vertices maintains the rigid circuit property. Conversely if $\mathscr{G}$ has a circuit with more than three vertices which has no chord, then no

1 All graphs considered in this section are finite and undirected, and have no multiple edges or loops.

2 This simple test has apparently not been noted before. See [7], where a more complicated test is used. 
vertex of this circuit can be deleted in the process.

Figures 7.1 and 7.2 show examples of rigid circuit graphs, the vertices being numbered in such a way that successively suppressing the next vertex in the numbering fulfills the test.

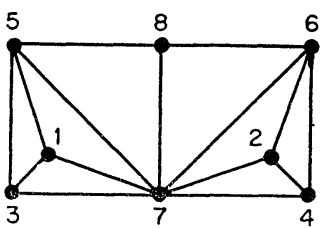

Fig. 7.1

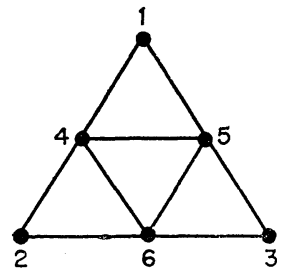

Fig. 7.2

We shall return to rigid circuit graphs in a moment, but at this point we need to introduce a certain incidence matrix which can be associated with an arbitrary graph $\mathscr{G}$. We call this matrix the dominant clique vs. vertex matrix. As will be obvious, it specifies $\mathscr{G}$ completely. Let $\mathscr{G}$ be any graph. We can consider the family of all sets of vertices which form cliques in $\mathscr{G}$ and partially order these by set inclusion. The maximal elements in this partial ordering will be termed the dominant cliques of $\mathscr{G}$. Since two vertices of $\mathscr{G}$ are joined by an edge if and only if they belong to some dominant clique, the dominant clique vs. vertex incidence matrix characterizes $\mathscr{G}$.

Such incidence matrices for the graphs of Figs. 7.1 and 7.2 are shown in Figs. 7.3 and 7.4, respectively.

$$
\left[\begin{array}{llllllll}
1 & 2 & 3 & 4 & 5 & 6 & 7 & 8 \\
1 & 0 & 1 & 0 & 1 & 0 & 1 & 0 \\
0 & 1 & 0 & 1 & 0 & 1 & 1 & 0 \\
0 & 0 & 0 & 0 & 1 & 0 & 1 & 1 \\
0 & 0 & 0 & 0 & 0 & 1 & 1 & 1
\end{array}\right]
$$

Fig. 7.3

$\left.\begin{array}{llllll}1 & 2 & 3 & 4 & 5 & 6 \\ 1 & 0 & 0 & 1 & 1 & 0 \\ 0 & 1 & 0 & 1 & 0 & 1 \\ 0 & 0 & 1 & 0 & 1 & 1 \\ 0 & 0 & 0 & 1 & 1 & 1\end{array}\right]$

Fig. 7.4

For rigid circuit graphs, the determination of the dominant cliques can be carried out in conjunction with the test for the rigid circuit property described above. Upon deleting a simplicial vertex, simply list it together with its neighbors in the reduced graph. If the original graph $\mathscr{G}$ has $n$ vertices, this yields a set of $n$ cliques. The dominant ones of these $n$ cliques are the dominant cliques of $\mathscr{G}$. For these are certainly cliques of $\mathscr{G}$. Moreover, if $\mathscr{C}$ is an arbitrary clique in $\mathscr{G}$, consider the first vertex of $\mathscr{C}$ which is deleted in the process. At this stage we list a clique $\mathscr{C}^{\prime}$ that includes $\mathscr{C}$.

The theory and methods we have developed for studying the consecutive 1's property can now be applied to interval graphs via Theorem 7.1 below. 
THEOREM 7.1. A graph $\mathscr{G}$ is an interval graph if and only if the dominant clique vs. vertex incidence matrix of $\mathscr{G}$ has the consecutive 1's property.

Proof. Let $D$ be the dominant clique vs. vertex incidence matrix of $\mathscr{G}$, so arranged that the 1 's in each column occur consecutively. Suppose the first and last 1's in the $j$-th column of $D$ occur in positions $f_{j}$ and $l_{j}$, respectively. Then $\mathscr{G}$ is the intersection graph of the intervals $\left[f_{j}-1, l_{j}\right], j=1,2, \cdots, n$.

Conversely, let $\mathscr{G}$ be the intersection graph of a set of $n$ (closed) intervals $I_{j}, j=1,2, \cdots, n$. Let the distinct endpoints $e_{1}, e_{2}, \cdots, e_{m}$ of these intervals be ordered so that $e_{1}<e_{2}<\cdots<e_{m}$. Then the $m$ by $n$ incidence matrix $A=\left(a_{i j}\right)$, defined by setting $a_{i j}=1$ or 0 according as $e_{i}$ is or is not in $I_{j}$, has consecutive 1 's in each column. Moreover, $D$ is obtained from $A$ by deleting rows. Hence $D$ has the consecutive 1 's property.

The incidence matrix of Fig. 7.3 has the consecutive 1's property; that of Fig. 7.4 does not. (A rearranged form of Fig. 7.3 is shown in Fig. 7.5). Consequently the graph of Fig. 7.1 is an interval graph (its "intervals" being displayed in Fig. 7.5), but that of Fig. 7.2 is not.

$$
\left.\begin{array}{llllllll}
1 & 2 & 3 & 4 & 5 & 6 & 7 & 8 \\
1 & 0 & 1 & 0 & 1 & 0 & 1 & 0 \\
0 & 0 & 0 & 0 & 1 & 0 & 1 & 1 \\
0 & 0 & 0 & 0 & 0 & 1 & 1 & 1 \\
0 & 1 & 0 & 1 & 0 & 1 & 1 & 0
\end{array}\right]
$$

Fig. 7.5

8. Consecutive 1's and total unimodularity. $A(0,1)$-matrix $A$ is said to be totally unimodular [6] if each of its square submatrices has determinant \pm 1 or 0 . It follows from known sufficient conditions for the total unimodularity property that if $A$ has the consecutive 1's property, then $A$ is totally unimodular [6]. This fact can also be proved directly without difficulty by induction on the number of rows in a square matrix having the consecutive 1's property. The total unimodularity property has significant implications in linear inequality theory. In particular, if $A$ is totally unimodular, and if $b$ is a vector having integral components, then the convex polyhedron defined by the linear inequalities

$$
\begin{gathered}
A u \geqq b, \\
u \geqq 0
\end{gathered}
$$

has all integral extreme points. That is, if $u$ is an extreme solution 
of (8.1), then $u$ has integral components. Consequently, the linear program

$$
\begin{aligned}
& A u \geqq b, \\
& u \geqq 0, \\
& \text { minimize } c \cdot u
\end{aligned}
$$

always has integral solution vectors $u$, provided $A$ is totally unimodular and $b$ is integral. In (8.2), $c$ is a given real vector.

We can apply these facts and Theorem 7.1 to derive a certain combinatorial duality theorem for interval graphs which, though similar in some respects to known results about rigid circuit graphs [2, 4], does not hold for the latter. To state this theorem, we first require a definition. We shall say that a set of vertices in a graph $\mathscr{G}$ represents all dominant cliques of $\mathscr{G}$ provided each dominant clique contains some vertex of the set. Our interest is in a minimum representation, that is, a set of vertices which represents all dominant cliques and has minimum cardinality over all such sets.

THEOREM 8.1. Let $\mathscr{G}$ be an interval graph. Then the minimum number of vertices of $\mathscr{G}$ required to represent all dominant cliques is equal to the maximum number of dominant cliques that are mutually disjoint.

Proof. Let $D$ be the dominant clique vs. vertex incidence matrix of $\mathscr{G}$. In view of Theorem 7.1 and the preceding discussion, the problem of finding a minimum representation of dominant cliques can be posed as that of solving the linear program

$$
\begin{aligned}
& D u \geqq 1, \\
& u \geqq 0, \\
& \text { minimize } 1 \cdot u .
\end{aligned}
$$

Here 1 is a vector all of whose components are unity. The dual of (8.3) is

$$
\begin{aligned}
& D^{T} w \leqq 1, \\
& \quad w \geqq 0, \\
& \operatorname{maximize} 1 \cdot w .
\end{aligned}
$$

Since $D$ is totally unimodular, so is $D^{T}$, and thus the program (8.4) also has $(0,1)$-solution vectors $w$. Consequently (8.4) asks for the maximum number of dominant cliques that are pairwise disjoint. Thus Theorem 8.1 follows from the duality theorem for linear inequalities. It can be seen similarly that if $\mathscr{G}$ is an interval graph, then the 
maximum number of vertices, no two of which are in the same dominant clique (i.e., the maximum number of vertices, no two of which are joined by an edge) is equal to the minimum number of dominant cliques that cover all vertices (i.e., the minimum number of cliques that cover all vertices). This theorem is in fact known to be true more generally for rigid circuit graphs [2,4]. But Theorem 8.1 is false for rigid circuit graphs. The graph of Fig. 7.2 is a case in point, since the minimum number of vertices required to represent all dominant cliques is two, whereas the maximum number of mutually disjoint dominant cliques is one.

\section{REFERENCES}

1. S. Benzer, On the topology of the genetic fine structure, Proc. Nat. Acad. Sci. U.S.A. 45 (1959), 1607.

2. G. A. Dirac, On rigid circuit graphs, Abhandlungen Mathematischen Seminar Universität Hamburg 25 (1961), 72.

3. P. C. Gilmore and A. J. Hoffman, A characterization of comparability graphs and of interval graphs, in Can. J. Math. 16 (1964), 539.

4. A. Hajnal and J. Suranyi, Über die Auflösung von Graphen in vollständige Teilgraphen, Ann. Univ. Sci. Budapest, Sect. Math. 1 (1958), 113.

5. G. Hajos, Über eine Art von Graphen, Internationale Math. Nachrichten, 11 (1957), 65.

6. A. J. Hoffman and J. B. Kruskal, Integral boundary points of convex polyhedra, Annals of Math. Study 38, Princeton University Press, Princeton, New Jersey, (1956), 223.

7. C. G. Lekkerkerker and J. Ch. Boland, Representation of a finite graph by a set of intervals on the real line, Fund. Math. Polska Akad. Nauk, 51 (1962), 45. 



\section{PACIFIC JOURNAL OF MATHEMATICS}

\section{EDITORS}

\author{
H. Samelson \\ Stanford University \\ Stanford, California \\ R. M. Blumenthal \\ University of Washington \\ Seattle, Washington 98105
}

\author{
J. Dugundu \\ University of Southern California \\ Los Angeles, California 90007 \\ *Richard Arens \\ University of California \\ Los Angeles, California 90024
}

\section{ASSOCIATE EDITORS}
E. F. BeCKENBACH
B. H. NeUMANN
F. WOLF
K. YosidA

\section{SUPPORTING INSTITUTIONS}

\author{
UNIVERSITY OF BRITISH COLUMBIA \\ CALIFORNIA INSTITUTE OF TECHNOLOGY \\ UNIVERSITY OF CALIFORNIA \\ MONTANA STATE UNIVERSITY \\ UNIVERSITY OF NEVADA \\ NEW MEXICO STATE UNIVERSITY \\ OREGON STATE UNIVERSITY \\ UNIVERSITY OF OREGON \\ OSAKA UNIVERSITY \\ UNIVERSITY OF SOUTHERN CALIFORNIA
}

\author{
STANFORD UNIVERSITY \\ UNIVERSITY OF TOKYO \\ UNIVERSITY OF UTAH \\ WASHINGTON STATE UNIVERSITY \\ UNIVERSITY OF WASHINGTON \\ * * * * \\ AMERICAN MATHEMATICAL SOCIETY \\ CALIFORNIA RESEARCH CORPORATION \\ SPACE TECHNOLOGY LABORATORIES \\ NAVAL ORDNANCE TEST STATION
}

Mathematical papers intended for publication in the Pacific Journal of Mathematics should by typewritten (double spaced). The first paragraph or two must be capable of being used separately as a synopsis of the entire paper. It should not contain references to the bibliography. No separate author's resumé is required. Manuscripts may be sent to any one of the four editors. All other communications to the editors should be addressed to the managing editor, Richard Arens, at the University of California, Los Angeles, California 90024.

50 reprints per author of each article are furnished free of charge; additional copies may be obtained at cost in multiples of 50 .

The Pacific Journal of Mathematics is published quarterly, in March, June, September, and December. Effective with Volume 13 the price per volume (4 numbers) is $\$ 18.00$; single issues, $\$ 5.00$. Special price for current issues to individual faculty members of supporting institutions and to individual members of the American Mathematical Society: $\$ 8.00$ per volume; single issues $\$ 2.50$. Back numbers are available.

Subscriptions, orders for back numbers, and changes of address should be sent to Pacific Journal of Mathematics, 103 Highland Boulevard, Berkeley 8, California.

Printed at Kokusai Bunken Insatsusha (International Academic Printing Co., Ltd.), No. 6, 2-chome, Fujimi-cho, Chiyoda-ku, Tokyo, Japan.

PUBLISHED BY PACIFIC JOURNAL OF MATHEMATICS, A NON-PROFIT CORPORATION

The Supporting Institutions listed above contribute to the cost of publication of this Journal, but they are not owners or publishers and have no responsibility for its content or policies.

* Basil Gordon, Acting Managing Editor until February 1, 1966. 


\section{Pacific Journal of Mathematics}

\section{Vol. 15, No. $3 \quad$ November, 1965}

David R. Arterburn and Robert James Whitley, Projections in the space of

bounded linear operators .................................

Robert McCallum Blumenthal, Joram Lindenstrauss and Robert Ralph Phelps,

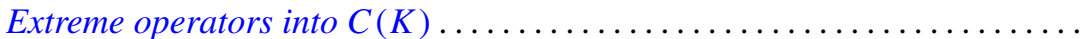

L. Carlitz, A note on multiple exponential sums ................... 757

Joseph A. Cima, A nonnormal Blaschke-quotient .................... 767

Paul Civin and Bertram Yood, Lie and Jordan structures in Banach algebras . . . 775

Luther Elic Claborn, Dedekind domains: Overrings and semi-prime

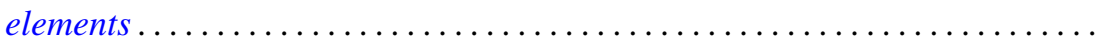

799

Luther Elic Claborn, Note generalizing a result of Samuel's .............. 805

George Bernard Dantzig, E. Eisenberg and Richard Warren Cottle, Symmetric

dual nonlinear programs ................................... 809

Philip J. Davis, Simple quadratures in the complex plane ............... 813

Edward Richard Fadell, On a coincidence theorem of F. B. Fuller ............ 825

Delbert Ray Fulkerson and Oliver Gross, Incidence matrices and interval

graphs ........................................ 835

Larry Charles Grove, Tensor products over $H^{*}$-algebras ..................

Deborah Tepper Haimo, $L^{2}$ expansions in terms of generalized heat polynomials

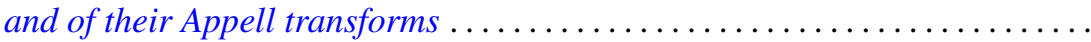

I. Martin (Irving) Isaacs and Donald Steven Passman, A chardcterization of groups in terms of the degrees of their characters ..........

Donald Gordon James, Integral invariants for vectors over local fields........ 905

Fred Krakowski, A remark on the lemma of Gauss ................... 917

Marvin David Marcus and H. Minc, A subdeterminant inequality ........... 921

Kevin Mor McCrimmon, Norms and noncommutative Jordan algebras ........ 925

Donald Earl Myers, Topologies for Laplace transform spaces ............... 957

Olav Njstad, On some classes of nearly open sets .................... 961

Milton Philip Olson, A characterization of conditional probability ........... 971

Barbara Osofsky, A counter-example to a lemma of Skornjakov .............. 985

Sidney Charles Port, Ratio limit theorems for Markov chains ............... 989

George A. Reid, A generalisation of $W^{*}$-algebras ...................... 1019

Robert Wells Ritchie, Classes of recursive functions based on Ackermann's

function ........................................... 1027

Thomas Lawrence Sherman, Properties of solutions of $n$th order linear

differential equations ........................................ 1045

Ernst Snapper, Inflation and deflation for all dimensions . .............. 1061

Kondagunta Sundaresan, On the strict and uniform convexity of certain Banach

spaces............................................. 1083

Frank J. Wagner, Maximal convex filters in a locally convex space .......... 1087

Joseph Albert Wolf, Translation-invariant function algebras on compact 